\title{
Immune-mediated inflammatory disease therapeutics: past, present and future
}

\section{Iain B. McInnes and Ellen M. Gravallese}

Abstract | Immune-mediated inflammatory diseases are common and clinically diverse. Although they are currently incurable, the therapeutic armamentarium for immune-mediated inflammatory diseases has been transformed in the past two decades. We have moved from the wide application of broad-spectrum immune modulators to the routine use of agents with exquisite specificity, arising from monoclonal and molecular biotechnology and more recently from highly targeted medicinal chemistry. Here we describe key advances and lessons that drove this remarkable progress and thereafter reflect on the next steps in this ongoing journey.

"It was the best of times, it was the worst of times, it was the age of wisdom...it was the epoch of incredulity, it was the season of Light, it was the season of Darkness, it was the spring of hope..." (Tale of Two Cities, Charles Dickens 1859).

Immune-mediated inflammatory diseases (IMIDs) comprise a common, clinically diverse group of conditions for which there are no current cures. They offer a remarkable paradigm for the successful application of modern molecular and computational techniques applied to immunological target discovery and consequent therapeutic development. They share common underlying pathogenetic features ('public' immune pathways), but also present unique ('private') pathways that define, for example, their clinical phenotype, age and sex distribution, tissue localization and therapeutic response profile. IMIDs include rheumatoid arthritis (RA), the spondyloarthritis ( $\mathrm{SpA}$ ) disease spectrum, connective tissue disorders, cutaneous inflammatory conditions (including psoriasis and atopic dermatitis), inflammatory bowel disease (IBD), asthma and autoimmune neurological diseases such as multiple sclerosis. Thus, they pose significant systemic medical challenges. Moreover, these diseases are often accompanied by various co-morbidities, including cardiovascular disease, metabolic and bone disorders and cognitive deficit, that further unfavourably impact quality of life and mortality. We focus here primarily on immune-mediated and autoimmune rheumatic diseases, as these offer a compelling example of the progress that has occurred in targeted disease therapy.

In the past two decades, the therapeutic armamentarium for IMIDs has been transformed. We have moved from the wide application of broad-spectrum immune modulators to the routine use of highly specific agents, arising from monoclonal and molecular biotechnology and latterly the application of highly targeted medicinal chemistry. We describe the key advances and lessons that drove the development of novel immune-targeted therapeutics, and also reflect on the next steps in this remarkable journey.

\section{Lessons from therapeutics past}

IMID therapeutics towards the end of the twentieth century relied heavily on glucocorticoids and a range of other small chemical entities that were largely borrowed from other disciplines on the basis of their antiproliferative or cell metabolism effects. From the 1940s, glucocorticoids served as a mainstay of therapy for various IMIDs. Although versatile and effective, they demonstrated diminishing therapeutic benefit over time, as well as substantial toxicity with regard to bone, cardiovascular systems and metabolic function. Other mainstay therapeutics included agents such as methotrexate, azathioprine, sodium aurothiomalate (gold salts), sulfasalazine, hydroxychloroquine, D-penicillamine and mycophenolate; however, their clinical application was rarely properly defined in terms of immune specificity, or indeed in terms of underlying disease pathogenesis ${ }^{1}$. These agents were used in sequence, or in combination, with considerable caution arising from a significant adverse event burden. Clinical outcomes were modest at best, as judged by modern ambitions - partial responder and non-responder populations were common, and remission was rarely achieved. Long-term disability was, sadly, the expected norm across IMIDs.

Two critical advances presaged a transformed landscape. A new paradigm of biotherapeutic innovation based on key pathogenic disease mechanisms was pioneered, initially with cytokine blockade and the groundbreaking application of tumour necrosis factor (TNF) inhibitors in the treatment of $\mathrm{RA}^{2,3}$. This brought about rapid changes in the advent of immune-targeted therapeutics, both biological and, more recently, small-molecule-based therapeutics, targeting a wide range of cytokines and their receptors, inflammatory cell trafficking pathways and cell regulatory ligand pairs, and the application of cell depletion strategies. This expanding diversity of biological and small-molecule therapies occurred alongside a second significant advance: the recognition that strict control of inflammation is critical to disease outcome. Substantial strategic changes thus occurred in the clinical approach to IMID management, with increased emphasis on early diagnosis and intervention, followed by the goal to achieve remission or low-disease activity states, while preventing target organ damage and thereby positively impacting both quality of life and longevity ${ }^{4,5}$.

\section{Importance of pathogenic disease} mechanisms. Many important lessons were learnt in the early days of biological drug development for IMIDs (FIG. 1). Cell depletion approaches, for example using 
monoclonal antibodies directed against T cells (specific for CD3, CD4 or CDw52 (alemtuzumab)), showed promise in open-label studies, but in controlled trial designs these approaches consistently failed $^{6,7}$, in contrast to experiences in the transplantation and cancer fields. This may have been due in part to the failure to deplete relevant $\mathrm{T}$ cells within inflamed sites such as synovial tissues, with emergence of dominant clonotypes, as was demonstrated in trials in RA with alemtuzumab ${ }^{8,9}$, or to the depletion of protective regulatory T cell subsets. Such results prompted a re-evaluation of pathogenesis models, in particular concerning the predominance of adaptive immune mechanisms and functionally discrete $\mathrm{T}$ cell subsets in driving the established phase of IMIDs. In parallel, a rethinking of trial design, with meticulous attention to development of novel composite outcome measures, permitted refined evaluation of clinical efficacy in IMIDs. Thereafter, the notion, arising from earlier studies in sepsis, that functional redundancy in cytokine biology would obviate the success of targeted immune interventions against specific cytokines, was utterly refuted in the clinic. This is best exemplified in the application of TNF and IL-1 inhibitors in RA and shortly thereafter in IBD $^{2,3,10}$. Whereas in the 1990s the perceived immune functional profile of the TNF and IL-1 pathways was similar (both cytokines were present in disease tissue and appeared viable as targets in in vivo disease models), TNF inhibitors, but not IL-1 inhibitors, were highly successful in clinical application in RA. These results demonstrated that, although IL-1 is a cytokine of functional importance in animal models of RA, its role in perpetuating inflammation is less critical than that of TNF in human RA, and that targeting TNF alone is a highly effective approach. The idea that IL-1 was an inferior therapeutic target that lacked hierarchical dominance was later refuted when IL-1 inhibitors were successfully developed to treat monogenic autoinflammatory disorders such as Muckle-Wells syndrome and, thereafter, inflammasome-driven diseases such as gout ${ }^{11,12}$. A core pathogenic role for IL-1 in these latter conditions is now well understood. Thus, the crucial importance of a highly evolved understanding of pathogenic mechanisms of IMIDs was established. Paradoxically, midst this success, the relative weaknesses in our preclinical discovery paradigms and in particular our core immunological understanding of many IMIDs were also exposed.

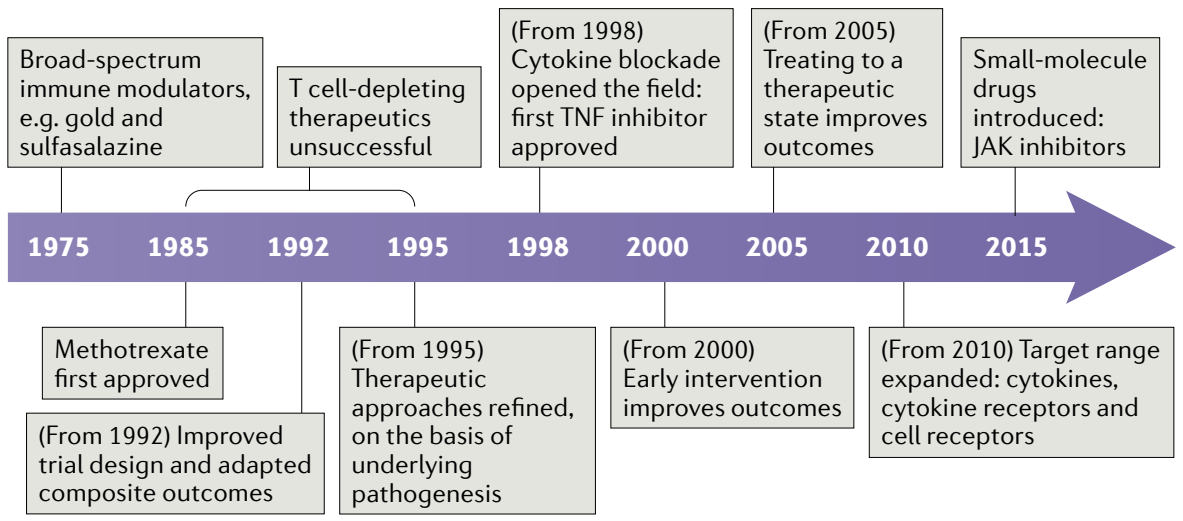

Fig. 1 | Timeline of therapeutic strategies for immune-mediated inflammatory diseases. This timeline highlights the key lessons learnt over the past 40 years that led to the development of immune therapeutics, from broad spectrum to highly specific, for immune-mediated inflammatory diseases ${ }^{59}$. JAK, Janus kinase; TNF, tumour necrosis factor.

Notwithstanding the foregoing, the recognition of public and private hierarchical immune pathways driving pathogenesis across IMIDs arose. The concept of public pathways was clear in the application of TNF inhibitors across a range of disorders, including axial SpA, IBD, psoriasis and psoriatic arthritis (PsA) (see, for example, REFS $^{13,14}$ ). Once again, however, lessons were learnt from the failures of this strategy, and the existence of private pathways of disease was also recognized. For example, whereas IL-6 receptor and IL-6 inhibition were successful in RA and vasculitis, this approach failed in psoriasis and axial SpA. IL-17A inhibition was efficacious in psoriasis, PsA and axial SpA, but not in RA nor IBD (see, for example, REFS ${ }^{15-17}$ ). Indeed, IL-17A inhibition may result in disease exacerbation in IBD, informing an important barrier function in the gut, which is independent of local IL-23p19 expression, as inhibition of IL-23p19 is more likely anti-inflammatory ${ }^{18}$. Such studies in turn provoked notions of a hierarchy of diseases defined by central immune pathways - a molecular taxonomy of sorts for IMIDs ${ }^{19}$.

Importance of controlling inflammation and immune activation. In parallel with lessons learnt regarding specific targeting of immune pathways in IMIDs, it was recognized that the best disease outcomes occur when inflammation is completely controlled. The field thus moved to early and aggressive use of targeted therapies to control inflammation ${ }^{5,20}$. Such an approach prevents irreversible tissue damage, as has been demonstrated in the tight control of inflammation in RA and protection from the subsequent destruction of joint cartilage and bone. Indeed, it has also become evident that when tissue damage does occur, such as erosion of the articular bone seen in RA, repair of the damaged bone is possible, but only in settings in which inflammation is rigorously controlled ${ }^{21}$.

The current state of the art in IMID therapeutics thus seeks high response rates and remission for a significant proportion of patients, achieved against an acceptable toxicity burden. These ambitious targets have been achieved to variable levels across the rheumatic IMIDs, but much unmet need remains. There is increasing recognition that IMIDs should be defined on the basis of their absolute immunological activity state, rather than on the level of disease activity relative to a prior timepoint (defined by transient response to an intervention). By this means, an IMID might be classified as active with no therapy, in a residual activation state despite (a range of) interventions or in remission, either drug-maintained or drug-free. Studies of drug-free and drug-maintained remission offer particular possibilities in terms of identifying immunological pathways associated with persistence of disease in the absence of clinical manifestations. For example, molecular analyses of 'cleared psoriasis' plaques after treatment with IL-17A inhibitors or TNF inhibitors do not reveal molecular normality, but rather demonstrate evidence of a healed state $^{22}$. Mucosal biopsy samples of resolved colonic lesions similarly exhibit pathways associated with a regulatory or 'restrained' state $^{23}$. With use of a similar approach, a subset of macrophages positive for tyrosineprotein kinase Mer (MERTK) and CD206 has been described in synovial biopsy samples from patients with RA in clinical remission that is associated with a long-term disease-free state ${ }^{24}$. 
A final key lesson has been the recognition that if IMIDs are controlled early and aggressively with achievement of immunological remission, we can begin to successfully reduce and, even in some cases, eliminate therapies in the short term $^{25}$. However, in most cases, disease will recur. Thus, a fundamental change in the underlying immunological process has not been fully realized; rather, the disease has been successfully suppressed. Earlier use of biological agents is more efficacious than late disease intervention. The reason for this is not yet clear but it could reflect a more tractable clinical state in early disease, with less target organ damage and therefore enhanced residual potential for recovery. It is, however, possible that this clinical observation speaks to an evolving immunological pathogenic predominance over time in IMIDs. It is well accepted that different immune pathways operate over the course of an infectious disease and perhaps, as organ damage accumulates, we should explore similar models in IMIDs over time, with sequential use of targeted therapeutics arising in consequence. Moreover, in terms of strategic intervention, there is a move from early intervention to the interception of disease at a phase of 'predisease. This is particularly exciting in the area of psoriasis, where approximately $30 \%$ of patients will go on to develop PsA and other related axial SpA phenomena and in whom, in theory, appropriate immune therapeutic intervention could be preventive. Similar options arise with the identification of 'pre-RA', which is loosely defined on the basis of genetic risk factors, family history and the presence of circulating autoantibodies recognizing altered post-transcriptionally modified peptides $^{26}$. There is particular interest in targeting adaptive immune mechanisms in this context as this is generally considered to be the time in disease evolution when such pathways predominate over innate or stromal cell contributions. Several clinical trials are ongoing or have recently been completed that address this by depleting B cells with rituximab or by checkpoint modulation with abatacept (targeting cytotoxic T lymphocyte-associated protein 4$)^{27,28}$. The long-term capacity to truly prevent RA development is an exciting prospect for the future and serves as a paradigm lead for the IMID field.

\section{Current therapeutics in IMIDs}

Biological drugs are now routinely used to treat a broad range of IMIDs; key pathways and diseases that have been effectively
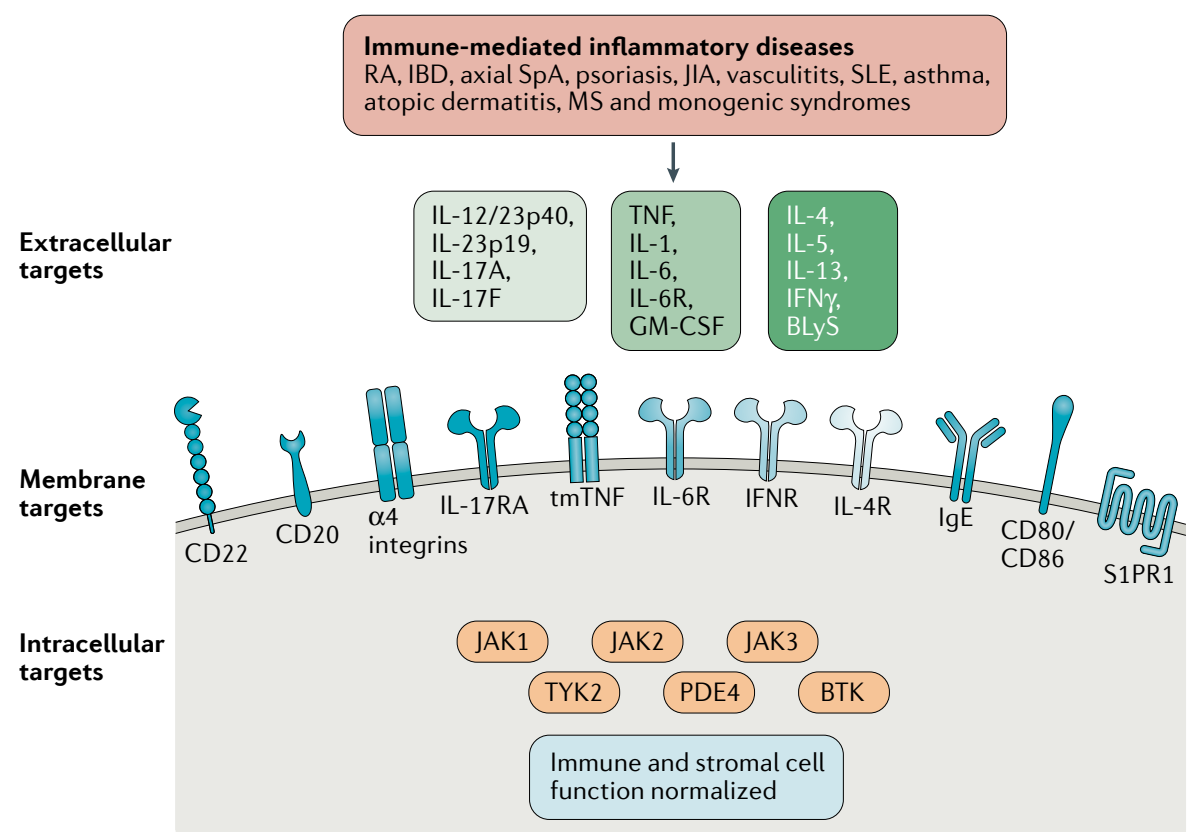

Fig. 2 | Key targets for the management of immune-mediated inflammatory diseases. A selection of the key extracellular, membrane-bound and intracellular targets for biological and small-molecule therapies that have driven the transformation in management of immune-mediated inflammatory diseases is shown ${ }^{60,61}$. BLyS, B lymphocyte stimulator; GM-CSF, granulocyte-macrophage colony-stimulating factor; IBD, inflammatory bowel disease; IFN $\gamma$, interferon- $\gamma$; IFNR, interferon receptor; JAK, Janus kinase; JIA, juvenile idiopathic arthritis; MS, multiple sclerosis; PDE4, phosphodiesterase 4; RA, rheumatoid arthritis; SLE, systemic lupus erythematosus; SpA, spondyloarthritis; S1PR1, sphingosine 1-phosphate receptor 1 ; tmTNF, transmembrane tumour necrosis factor.

targeted are summarized in FIG. 2. These biological drug targets include cytokines and their receptors, or cell subsets with a view to altered trafficking, modulation of cellular activation state or cellular depletion. Clinical benefits may accrue across a range of immune phenotypes. Thus, if defined, for example, on the basis of T cell subset dominance, type 1-, type 2 - and type 17-associated diseases have all been successfully targeted using relevant cognate interventions.

A further important advance has been the refined use of small-molecule inhibitors as IMID therapeutics. Small-molecule therapies were rarely developed on the basis of a firm pathogenic understanding of IMIDs. Now, however, the human kinome (representing the complete set of lipid and protein kinases encoded in the human genome) has proven particularly appealing for the discovery of targets for smallmolecule therapeutics. After several false dawns (for example, in targeting mitogenactivated protein kinase and tyrosineprotein kinase SYK), the introduction of Janus kinase (JAK) inhibitors represents interesting progress. Thus, a range of highly selective JAK inhibitors, variously directed against all JAKs or more selective for family members, particularly JAK1, are now approved for use in some IMIDs, with more indications expected soon. These agents are attractive because of their oral bioavailability, but even more so because of simultaneous combinatorial targeting of cytokine receptor effector function. With the use of these agents in several IMIDs, clinical trials are delivering response rates that are not inferior or are even superior to those achieved with biological agents, for the first time in a decade ${ }^{29,30}$.

There remains caution, however, in establishing that such broader immunosuppression (even assuming all effects are on target) can be delivered without some negative consequences, in particular with concern for toxicity affecting the cardiovascular system, host defence and cancer risk. In addition, some unexpected adverse events have been described, including thrombosis, that challenge our understanding of how JAK pathways contribute to interactions between the immune system and physiological functions ${ }^{30}$. Long-term observation of these potential interactions will be essential. Nonetheless, one key lesson is that with modern medicinal chemistry, including in silico and artificial intelligence-based applications of medicinal chemistry, it is possible to recreate, at least in part, the 
remarkable immune selectivity offered by biologicals and to place such interventions in the context of our understanding of disease pathogenesis. On the basis of both antiviral and broad-spectrum cytokine inhibitory properties, the JAK inhibitor baricitinib has potential to be successfully developed for use in the hyper-response phase of COVID-19 (REF. ${ }^{31}$ ).

Notably, the use of these drugs as 'molecular scalpels' has empowered mode-of-action studies, thereby driving reverse therapeutic to pathogenesis innovation. Elegant early studies examined the effects of TNF inhibitors in RA and elucidated a role for TNF in endothelial cell activation, leukocyte recruitment to target inflammatory tissue sites, promotion of the acute phase response and promotion of nociception and cognitive dysfunction. These studies also facilitated examination of contrasting modes of action of TNF inhibitors and other drug classes in IBD. A paradoxical inefficacy of the TNF receptor-IgG1 fusion protein etanercept compared with monoclonal TNF inhibitors was seen in $\mathrm{IBD}^{32}$, perhaps based on differential effects on depletion of or apoptosis of mucosal lymphocyte subsets expressing membrane TNF. Subsequently, extensive studies of the mode of action of biological agents and JAK inhibitors have proffered invaluable insights into disease pathogenesis across diseases, and perceived hierarchies of immune pathways that drive damage in distinct tissues have emerged $^{3,22,23,33}$. Imaging, high-sensitivity molecular analysis of cell types in peripheral blood and tissue biopsy approaches have elucidated fundamental biological immune effector pathways in IMIDs. Also informative has been the analysis of biopsy samples, in particular obtained before and after drug administration, using a range of cellular imaging and molecular resolution methods, including cytometry by time-of-flight microscopy, single-cell RNA sequencing and spatial transcriptomics. By these means, an iterative cycle of positive discovery is instated whereby successful and unsuccessful therapeutics can inform more efficient future drug development, supported by improved understanding of the human immune system.

An additional intriguing observation concerns discrete, differential responses across involved tissues, even in individual patients. For example, in the axial SpA spectrum, TNF inhibitors, IL-17A inhibitors and IL-23 inhibitors each deliver responses in skin, synovium and entheses. However, IL-17A and IL-23 inhibitors are superior in their magnitude of response in the skin, routinely delivering disease clearance in more than $50 \%$ of patients $\mathrm{s}^{34,35}$. TNF and IL-23 inhibitors appear more efficacious in managing inflammation of the gastrointestinal tract, whereas IL-17A inhibitors are ineffective or even deleterious $^{36}$. TNF and IL-17A inhibitors, but not IL-23 inhibitors, are effective in reducing inflammation in the axial skeleton ${ }^{37}$. These differences in response may be the result of unique cytokines that perpetuate these distinct disease states. However, one fascinating alternative possibility arises - namely, that the target tissue itself is the dominant arbiter of disease pathogenesis, due to tissue-specific stromal elements with which inflammatory cells interact, and that the target tissue may even define the dominant immune effector pathways that drive local disease against a common antecedent genome, epigenome, microbiome and 'envirome'.

\section{Insights from adverse effects}

In general, specific immune modifiers increase the overall risk of infection over time (odds ratio 1.5) ${ }^{38,39}$. Intriguingly the distinct modes of IMID therapeutic intervention elicit discrete adverse effects, thereby providing 'proof-of-pathway' involvement in host defence against various infections. These adverse effects constitute relative contraindications in clinical practice. Some, but not all, of these adverse effects correspond to vulnerabilities predicted on the basis of immunodeficiency studies and should be combined with these immunodeficiency studies to map essential host defence networks ${ }^{40}$. For example, TNF inhibitors predispose to reactivation of mycobacterial infection, highlighting the crucial role of TNF in maintenance of human granulomas and host defence against mycobacteria $^{41}$. IL-17A inhibitors elicit an expected increased risk of fungal infections, but not invasive fungal disease, for reasons that are not yet clear. Close observation of broader IL-17 cytokine family inhibition will be essential, such as in ongoing studies of dual inhibition of IL-17A and IL-17F ${ }^{42}$.

The use of JAK inhibitors is associated with a particular risk of shingles, which indicates the key role played by JAK-dependent pathways in certain cell subsets, particularly $\gamma$-chain-dependent natural killer cells and $\mathrm{CD}^{+} \mathrm{T}$ cells, for prevention of varicella zoster virus reactivation ${ }^{30}$.

Similarly, experimental medicine studies of IMID therapeutics now describe a greater integration of immune function and host physiological pathways than previously appreciated, as illustrated by the observed cardiovascular and metabolic co-morbidities in IMIDs. Accordingly, modification of inflammation alters levels of circulating lipids and other metabolic vascular risk factors. The remarkable specificity of biologicals and JAK inhibitors has now mapped these pathways in humans - this is particularly useful, as animal models generally poorly reflect these interactions. For example, IL-6R and JAK inhibitors both elicit rapid increases in, and altered protein composition of, circulating low-density, high-density and very-low-density lipoprotein cholesterol particles, indicative of fundamental roles for these immune pathways in regulating hepatic and peripheral cholesterol metabolism $^{33,43}$. TNF inhibitors similarly alter insulin sensitivity in patients with IMIDs. It is notable that recent Mendelian randomization studies now causally implicate metabolic dysfunction in the onset of immune-mediated disease - moreover, the unidirectionality of such relationships should not be assumed ${ }^{44}$. Recent magnetic resonance imaging and positron emission tomography-computed tomography studies demonstrate the impact of immune targeting on vascular inflammation in psoriasis, likely reflecting accelerated atherosclerosis in the context of systemic inflammation ${ }^{45}$. This provides indirect support for the rapidly expanding field of immune intervention in the management of cardiovascular risk, as exemplified by the Canakinumab, Anti-inflammatory Thrombosis Outcomes Study (CANTOS) ${ }^{46}$, in which blockade of IL- $1 \beta$ in patients with a history of myocardial infarction and evidence of ongoing inflammation resulted in a significantly lower rate of recurrent cardiovascular events than in placebotreated patients. Finally, attention has focused recently on the impact of biological interventions on cognitive function using magnetic resonance imaging of the brain before and after administration of biological therapies, providing direct evidence for crosstalk between immunological and neurological pathways in the context of chronic inflammation ${ }^{47}$. The idea that this may be generalizable to wider cognitive disorders is enticing.

\section{Future perspectives in IMID therapeutics}

There remain many unmet clinical needs across the IMIDs - in particular, remission is not yet achieved in sufficient numbers and long-term drug-free remission, tantamount to cure, is rarely achieved. Restoring immune dysregulation to normality is 
not yet possible. Furthermore, although there is evidence for relief of some disease manifestations beyond inflammation in IMIDs, such as fatigue and sleep disturbance, in the setting of successful biological therapies, pain pathways appear to be distinct from those of inflammation, and pain can persist despite adequate control of inflammation. There are currently a rather broad range of targets already in clinical trials. Further interrogation of the kinome, including targeting TYK2 and IRAK4, offers promise. Cellular therapeutics are also subject to extensive exploration, such as the use of stem cells, modified regulatory $\mathrm{T}$ cells, chimeric antigen receptor $\mathrm{T}$ cells and tolerized dendritic cells ${ }^{48,49}$. Imaginative approaches based on interactions between the immune and neurological systems using biostimulation are in phase II trials in RA, with potential across other IMIDs ${ }^{50}$. We highlight the following principles that should drive progress (FIG. 3).

New targets. The increasingly sophisticated application of state-of-the-art tissue analysis applied to imaging-guided biopsies of involved tissues is revealing novel targets for IMIDs. In particular, accessible biopsies in psoriasis, atopic dermatitis, IBD and inflammatory synovial disorders not only allow for mechanism-of-action studies as described earlier but also increasingly for the recognition of molecular phenotypes of disease, known as 'pathotypes', which may lead to novel therapeutic intervention. Such approaches, when combined with powerful bioinformatic approaches, can reveal unexpected molecules or cell subsets as targets. Single-cell RNA sequencing approaches also present the possibility of discovering rare but functionally important cell subsets. The description of PD $1{ }^{\text {hi }} \mathrm{CXCR} 5^{-}$peripheral T helper cells capable of B cell help in non-lymphoid tissues in RA makes such a case elegantly and offers interesting therapeutic possibilities as a result ${ }^{51}$.

Single-cell analyses of tissue biopsy samples have also directed our attention to non-professional immune cells, especially cells within the tissue stroma. Whereas targeting of the endothelium, adhesion molecules and angiogenesis in particular has been long examined, more recent studies make a persuasive case for targeting stromal (fibroblast) subsets in IMIDs. Several studies have defined stromal cell subsets, separable on the basis of consistent membrane markers, such as THY1 membrane glycoprotein, fibroblast activation protein- $\alpha$ and podoplanin, that exhibit discrete functional roles in the context of tissue inflammation. In vivo targeting of these cells in animal models of disease is encouraging ${ }^{52}$. Could overlooking the stromal compartment in prior studies explain the 'glass ceiling' of therapeutic response that still manifests itself in many IMIDs as partial response to targeted immune intervention? This would be consistent with an integrated tissue immune response model whereby in chronic disease the normal synchronous regulation and sequential occurrence of activator and then regulator pathways in the immune system are lost, or are at least asynchronous, under the influence of pervasive stromal pathways. These stromal pathways, responding perhaps to epigenetic modulators, including

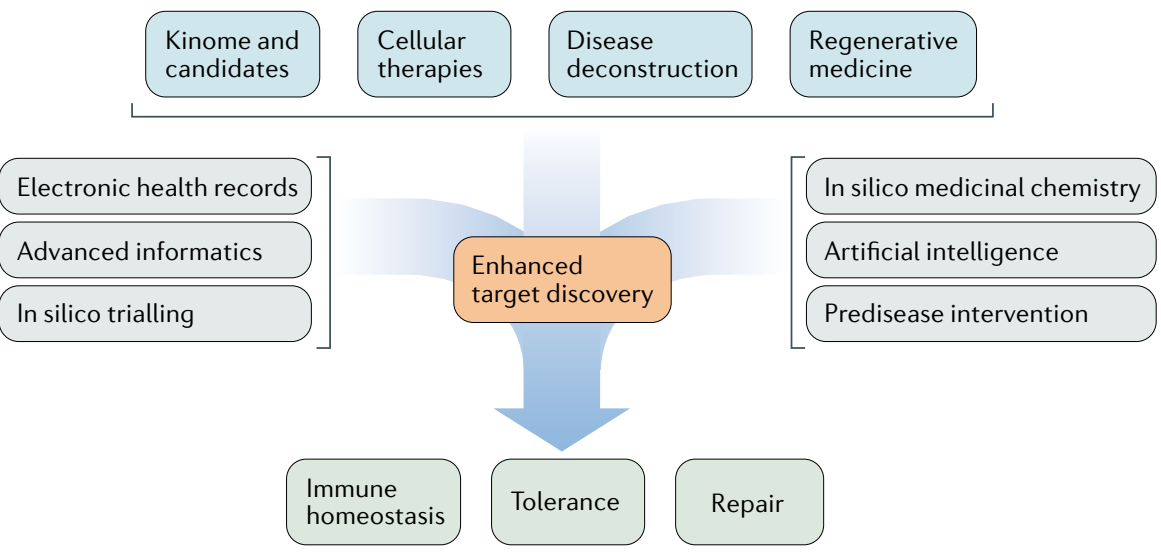

Fig. 3 | Future approaches for immune-mediated inflammatory disease therapeutics. We envisage future therapeutic design for immune-mediated inflammatory diseases to involve a combination of routes for candidate target discovery: kinome interrogation, novel cellular therapies, polyomic disease deconstruction and advances in regenerative medicine. In turn, this discovery programme will be enhanced by the application of artificial intelligence-based methods, in silico medicinal chemistry and in silico trialling, leading to immune homeostasis, immune tolerance and repair of organ damage.

hypoxia, recurrent cytokine exposure or persistent damage, thereby become the drivers of disease and initiators of disease recurrence. Intriguing recent evidence in patients with $\mathrm{RA}$ of $\mathrm{CD} 45^{-} \mathrm{CD} 31^{-} \mathrm{PDPN}^{+}$ pre-inflammatory mesenchymal cells, the appearance of which in peripheral blood immediately precedes clinical flare, would be consistent with such a model ${ }^{53}$.

New strategies. Just as the initial IMID revolution was supported by altered strategic approaches, so too will be the next phase, the phase of molecular revolution, which will capitalize on the application of (epi)genomic, transcriptomic, proteomic and metabolomic methods. Such methods are being extensively tested for their ability to predict the natural history of disease, optimal therapeutic selection and likely adverse event profiles for individuals with IMIDs. Early progress is being made through analysis of blood and especially through tissue-based approaches in RA, psoriasis and $\mathrm{IBD}^{54}$. Thus far, we lack critical validation of this molecular approach but are optimistic of its long-term success.

A further strategic advance will concern the adoption of strategies that induce remission and then maintenance, which may arise from the identification of immune or stromal pathways associated with clinical remission states in IMIDs. This approach is considered the standard of care in oncology yet has been less embraced in IMID therapeutics; perhaps the increasing range of available mechanisms of action and formal association of certain mechanisms with identifiable clinical states and immunological status will be permissive in this regard. Do we yet have the confidence to develop immune-targeting medicines that will work 'only' in a remission/maintenance context? A significant challenge remains the reliable assessment of immune states an 'immunological stethoscope' - the development of which would be an exciting advance. A final consequence of this high-fidelity resolution of disease pathogenesis will be to underpin future rational combinations of immune-targeted therapeutics in IMIDs.

As we move increasingly to electronic health records, the era of 'big data' in health and associated assembly of large biobanks, further potential lies in the comparisons that are now possible across IMIDs. There are now large, well-characterized biobanks for IMIDs that are ripe for comparative analysis to better define those immune pathways that are commonly engaged, or are lacking in the case of regulators, across IMIDs and 
those that may offer more disease-specific potential. As an example, the IMID-Bio-UK metaconsortium (see 'Related links'), which includes data from diseases such as RA, PsA, psoriasis, systemic lupus erythematosus, Sjögren syndrome, primary biliary cholangitis and autoimmune hepatitis, confers such potential. Interrogation of data from such metaconsortia will allow the discovery or validation of identified pathways before embarking on clinical trials. It is encouraging that even clinical trials are rapidly evolving to include extensive in silico testing of new agents before clinical introduction ${ }^{55}$. In parallel, the creation of biobanks associated with phase II/III clinical trials should allow greater mechanistic interrogation of such clinical cohorts to offer insights as well as clinical efficacy data.

\section{Tolerance induction and other novel} approaches. The 'holy grail' in the IMID field remains the restoration of immune homeostasis. It is unclear whether this will be possible on the basis of our current understanding of the underlying network dysfunction in IMIDs that sustains the breach of tolerance, especially in the context of existing tissue damage. Numerous interesting approaches are focused particularly on expanding regulatory $\mathrm{T}$ cell subsets (such as with IL-2), introducing tolerogenic dendritic cells and other approaches ${ }^{48,49}$. Another emerging avenue is the use of chimeric antigen receptor $\mathrm{T}$ cells directed against $\mathrm{B}$ cells in autoantibody-producing diseases such as systemic lupus erythematosus ${ }^{56}$. In addition, myeloablative autologous stem cell transplantation has been used effectively for fatal diseases such as systemic sclerosis $^{57}$ and may be useful in other IMIDs. A further intriguing opportunity lies in characterization of the molecular pathways that underpin the development of immunerelated adverse events following checkpoint inhibitor administration for cancer. This provides a 'natural experiment' in which regulatory checkpoints are bypassed and subsequent self-tissue damage and the mechanisms involved can be evaluated in real time, with the onset of insult precisely $\mathrm{known}^{58}$. Finally, as the upstream pathways that drive IMIDs are increasingly recognized (for example, with roles for the microbiome and for smoking in pulmonary lesions in RA), there will be more opportunities for preventative approaches.

\section{Concluding remarks}

IMID therapeutics have been transformed - from the worst of times to the best of times - representing a remarkable success story for the application of modern molecular medicine driven by an ambitious and increasingly integrated experimental medicine field. However, we still do not achieve remission in sufficient numbers of patients with IMIDs, organ integrity is not always preserved and tissue repair is not typically achieved. Quality of life remains suboptimal for many patients, especially for those with complex rheumatic diseases and those with neurological IMIDs. Although the past two decades have brought remarkable progress and many lessons of value, perhaps in the search for curative therapeutics, we remain in 'the spring of hope'.

Iain B. McInnes (D) ${ }^{\prime \otimes}$ and Ellen M. Gravallese $e^{2,3}$

'College of Medical Veterinary and Life Sciences, University of Glasgow, Glasgow, UK.

${ }^{2}$ Division of Rheumatology, Inflammation, and Immunity, Department of Medicine, Brigham and Women's Hospital, Boston, MA, USA.

${ }^{3}$ Harvard Medical School, Boston, MA, USA.

凶e-mail: iain.mcinnes@glasgow.ac.uk

https://doi.org/10.1038/s41577-021-00603-1

Published online 13 September 2021

1. Kerrigan, S. A. \& Mclnnes, I. B. Reflections on 'older' drugs: learning new lessons in rheumatology. Nat. Rev. Rheumatol. 16, 179-183 (2020).

2. Maini, R. et al. Infliximab (chimeric anti-tumour necrosis factor alpha monoclonal antibody) versus placebo in rheumatoid arthritis patients receiving concomitant methotrexate: a randomised phase III trial. Lancet 354, 1932-1939 (1999).

3. Feldmann, M., Brennan, F. M. \& Maini, R. N. Rheumatoid arthritis. Cell 85, 307-310 (1996).

4. Combe, B. et al. 2016 update of the EULAR recommendations for the management of early arthritis. Ann. Rheum. Dis. 76, 948-959 (2017).

5. Smolen, J. S. et al. Treating rheumatoid arthritis to target: recommendations of an international task force. Ann. Rheum. Dis. 69, 631-637 (2010).

6. Burmester, G. R. \& Emmrich, F. Anti-CD4 therapy in rheumatoid arthritis. Clin. Exp. Rheumatol. 2 , 627-642 (1993)

7. Isaacs, J. D. et al. CAMPATH-1H in rheumatoid arthritis - an intravenous dose-ranging study. Br. J. Rheumatol. 35, 231-240 (1996).

8. Jendro, M. C., Ganten, T., Matteson, E., Weyand, C. $\&$ Goronzy, J. J. Emergence of oligoclonal T cell populations following therapeutic $T$ cell depletion in rheumatoid arthritis. Arthritis Rheum. 38, 1242-1251 (1995).

9. Ruderman, E. M., Weinblatt, M. E., Thurmond, L. M., Pinkus, G. M. \& Gravallese, E. M. Synovial tissue response to treatment with Campath 1-H. Arthritis Rheum. 38, 254-258 (1995).

10. Bresnihan, B. et al. Treatment of rheumatoid arthritis with recombinant human interleukin-1 receptor antagonist. Arthritis Rheum. 41, 2196-2204 (1998).

11. Booshehri, L. M. \& Hoffman, H. M. CAPS and NLRP3. J. Clin. Immunol. 39, 277-286 (2019).

12. Schlesinger, N. et al. Canakinumab for acute gouty arthritis in patients with limited treatment options: results from two randomised, multicentre, activecontrolled, double-blind trials and their initial extensions. Ann. Rheum. Dis. 71, 1839-1848 (2012)

13. Sandborn, W. J. et al. An engineered human antibody to TNF (CDP571) for active Crohn's disease: a randomized double-blind placebo-controlled trial. Gastroenterology 120, 1330-1338 (2001).

14. Reich, K. et al. Infliximab induction and maintenance therapy for moderate-to-severe psoriasis: a phase III, multicentre, double-blind trial. Lancet 366,1367-1374 (2005).

15. Gabay, C. et al. Tocilizumab monotherapy versus adalimumab monotherapy for treatment of rheumatoid arthritis (ADACTA): a randomised, double-blind, controlled phase 4 trial. Lancet 381 , 1541-1550 (2013).
16. Stone, J. H. et al. Trial of tocilizumab in giant-cell arteritis N. Engl. J. Med 377, 317-328 (2017).

17. McInnes, I. B. et al. Secukinumab, a human antiinterleukin-17A monoclonal antibody, in patients with psoriatic arthritis (FUTURE 2): a randomised, doubleblind, placebo-controlled, phase 3 trial. Lancet 386, 1137-1146 (2015).

18. Ma, C., Panaccione, R., Khanna, R., Feagan, B. G. \& Jairath, V. IL12/23 or selective IL23 inhibition for the management of moderate-to-severe Crohn's disease? Best Pract. Res. Clin. Gastroenterol. 38-39, 101604 (2019).

19. Schett, G., Elewaut, D., Mclnnes, I. B., Dayer, J. M. $\&$ Neurath, M. F. How cytokine networks fuel inflammation: toward a cytokine-based diseas taxonomy. Nat. Med. 19, 822-824 (2013).

20. Grigor, C. et al. Effect of a treatment strategy of tight control for rheumatoid arthritis (the TICORA study): a single-blind randomised controlled trial. Lancet 364 263-269 (2017)

21. Matzelle, M. et al. Resolution of inflammation induces osteoblast function and regulates the Wnt signaling pathway. Arthritis Rheum. 64, 1540-1550 (2012).

22. Hawkes, J. E., Chan, T. C. \& Krueger, J. G. Psoriasis pathogenesis and the development of novel targeted immune therapies. J. Allergy Clin. Immunol. 140, 645-653 (2017)

23. Neurath, M. F. \& Travis, S. P. Mucosal healing in inflammatory bowel diseases: a systematic review. Gut 61, 1619-1635 (2012).

24. Alivernini, S. et al. Distinct synovial tissue macrophage subsets regulate inflammation and remission in rheumatoid arthritis. Nat. Med. 26, 1295-1306 (2020).

25. Edwards, C. J., Fautrel, B., Schulze-Koops, H., Huizinga, T. W. J. \& Kruger, K. Dosing down with biologic therapies: a systematic review and clinicians' perspective. Rheumatology 56, 1847-1856 (2017).

26. Arend, W. P. \& Firestein, G. S. Pre-rheumatoid arthritis: predisposition and transition to clinical synovitis. Nat. Rev. Rheumatol. 8, 573-586 (2012).

27. Gerlag, D. M. et al. Effects of B-cell directed therapy on the preclinical stage of rheumatoid arthritis: the PRAIRI study. Ann. Rheum. Dis. 78, 179-185 (2019).

28. Al-Laith, M. et al. Arthritis prevention in the preclinical phase of RA with abatacept (the APIPPRA study): a multi-centre, randomised, double-blind, parallel-group, placebo-controlled clinical trial protocol. Trials 20, 429 (2019).

29. O'Shea, J. J. et al. The JAK-STAT pathway: impact on human disease and therapeutic intervention. Annu. Rev. Med. 66, 311-328 (2015).

30. Nash, P. et al. Points to consider for the treatment of immune-mediated inflammatory diseases with Janus kinase inhibitors: a consensus statement. Ann. Rheum. Dis. 80, 71-87 (2021).

31. Kalil, A. C. et al. Baricitinib plus remdesivir for hospitalized adults with Covid-19. N. Engl. J. Med. 384, 795-807 (2021).

32. Sandborn, W. J. et al. Etanercept for active Crohn's disease: randomized, double-blind, placebo-controlled trial Gastroenterology 121, 1088-1094 (2001).

33. McInnes, I. B. \& Schett, G. Pathogenetic insights from the treatment of rheumatoid arthritis. Lancet 389 , 2328-2337 (2017).

34. Langley, R. G. et al. Secukinumab in plaque psoriasis results of two phase 3 trials. N. Engl. J. Med. 371 , 326-338 (2014).

35. Reich, K. et al. Guselkumab versus secukinumab for the treatment of moderate-to-severe psoriasis (ECLIPSE): results from a phase 3, randomised controlled trial. Lancet 394, 831-839 (2019).

36. Hueber, W. et al. Secukinumab, a human anti-IL-17A monoclonal antibody, for moderate to severe Crohn's disease: unexpected results of a randomised, doubleblind placebo-controlled trial. Gut 61,1693-1700 (2012).

37. Siebert, S., Millar, N. L. \& McInnes, I. B. Why did IL-23p19 inhibition fail in AS: a tale of tissues, trials or translation? Ann. Rheum. Dis. 78, 1015-1018 (2019).

38. Sepriano, A. et al. Safety of synthetic and biological DMARDs: a systematic literature review informing the 2019 update of the EULAR recommendations for the management of rheumatoid arthritis. Ann. Rheum Dis. 79, 760-770 (2020)

39. Sbidian, E. et al. Systemic pharmacological treatments for chronic plaque psoriasis: a network meta-analysis. Cochrane Database Syst. Rev. 12, CD011535 (2021).

40. Casanova, J. L. \& Abel, L. Lethal infectious diseases as inborn errors of immunity: toward a synthesis of the 
germ and genetic theories. Annu. Rev. Pathol. 24 23-50 (2021)

41. Calabrese, C. \& Winthrop, K. L. Mycobacterial infections potentiated by biologics. Infect. Dis. Clin. North Am. 34, 413-423 (2020).

42. Ritchlin, C. T. et al. Bimekizumab in patients with active psoriatic arthritis: results from a 48-week, randomised, double-blind, placebo-controlled, dose-ranging phase $2 \mathrm{~b}$ trial. Lancet $395,427-440$ (2020).

43. McInnes, I. B. et al. Effect of interleukin-6 receptor blockade on surrogates of vascular risk in rheumatoid arthritis: MEASURE, a randomised, placebo-controlled study. Ann. Rheum. Dis. 74, 694-702 (2015).

44. Budu-Aggrey, A. et al. Evidence of a causal relationship between body mass index and psoriasis: a Mendelian randomization study. PLoS Med. 16, e1002739 (2019).

45. Aksentijevich, M., Lateef, S. S., Anzenberg, P., Dey, A. K. \& Mehta, N. N. Chronic inflammation, cardiometabolic diseases and effects of treatment: psoriasis as a human model. Trends Cardiovasc. Med. 30, 472-478 (2020).

46. Ridker, P. M. et al. Antiinflammatory therapy with canakinumab for atherosclerotic disease. N. Engl. J. Med. 377, 1119-1131 (2017).

47. Schrepf, A. et al. A multi-modal MRI study of the central response to inflammation in rheumatoid arthritis. Nat. Commun. 9, 2243 (2018).

48. Mosanya, C. H. \& Isaacs, J. D. Tolerising cellular therapies: what is their promise for autoimmune disease? Ann. Rheum. Dis. 78, 297-310 (2019).

49. Bluestone, J. A. \& Anderson, M. Tolerance in the age of immunotherapy. N. Engl. J. Med. 383, 1156-1166 (2020).

50. Koopman, F. A. et al. Vagus nerve stimulation inhibits cytokine production and attenuates disease severity in rheumatoid arthritis. Proc. Natl Acad. Sci. USA 113, 8284-8289 (2016).

51. Rao, D. A. et al. Pathologically expanded periphera $T$ helper cell subset drives B cells in rheumatoid arthritis. Nature 542, 110-114 (2017).

52. Croft, A. P. et al. Distinct fibroblast subsets drive inflammation and damage in arthritis. Nature $\mathbf{5 7 0}$ 246-251 (2019).

53. Orange, D. E. et al. RNA identification of PRIME cells predicting rheumatoid arthritis flares. N. Engl. J. Med. 383, 218-228 (2020).

54. Lliso-Ribera, G. et al. Synovial tissue signatures enhance clinical classification and prognostic/ treatment response algorithms in early inflammatory arthritis and predict requirement for subsequent biological therapy: results from the pathobiology of early arthritis cohort (PEAC). Ann. Rheum. Dis. 78 1642-1652 (2019).

55. Galindez, G. et al. Lessons from the COVID-19 pandemic for advancing computational drug repurposing strategies. Nat. Comput. Sci. 1, 33-41 (2021).

56. Kansal, R. et al. Sustained B cell depletion by CD19 targeted CAR T cells is a highly effective treatment for murine lupus. Sci. Transl. Med. 11, eaav1648 (2019).

57. Sullivan, K. M. et al. Myeloablative autologous stemcell transplantation for severe scleroderma. N. Engl. J. Med. 378, 35-47 (2018).

58. Postow, M. A., Sidlow, R. \& Hellmann, M. D Immune-related adverse events associated with immune checkpoint blockade. N. Engl. J. Med. 378 158-168 (2018)

59. Burmester, G. R., Bijlsma, J. W. J., Cutolo, M. \& Mclnnes, I. B. Managing rheumatic and musculoskeletal diseases - past, present and future Nat. Rev. Rheumatol. 13, 443-448 (2017).

60. Parulekar, A. D., Kao, C. C., Diamant, Z. \& Hanania, N. A. Targeting the interleukin- 4 and interleukin-13 pathways in severe asthma: current knowledge and future needs. Curr. Opin. Pulm. Med. 24, 50-55 (2018)

61. Burmester, G. R. et al. A randomised phase IIb study of mavrilimumab, a novel GM-CSR receptor alpha monoclonal antibody, in the treatment of rheumatoid arthritis. Ann. Rheum. Dis. 76, 1020-1030 (2017).

\section{Author contributions}

The authors contributed equally to all aspects of the article.

\section{Competing interests}

E.M.G. receives salary support from New England Journal of Medicine, royalties from UptoDate and the textbook Rheumatology and grant support from the US National Institutes of Health. I.B.M. has received honoraria and/or research funding from AbbVie, Amgen, AstraZeneca, Bristol Myers Squibb, Boehringer, Cabaletta Bio, Causeway Therapeutics, Compugen, Eli Lilly, Gilead, GlaxoSmithKline, Janssen, Novartis, Pfizer, Roche, Sanofi and UCB.

\section{Peer review information}

Nature Reviews Immunology thanks D. Rao, V. Strand and the other, anonymous, reviewer(s) for their contribution to the peer review of this work.

\section{Publisher's note}

Springer Nature remains neutral with regard to jurisdictional claims in published maps and institutional affiliations.

\section{RELATED LINKS}

IMID-Bio-UK metaconsortium: https://www.gla.ac.uk/research/ az/imid/

C) Springer Nature Limited 2021 